\title{
Intramolecular Oscillation of the Phosphorylation Domain of Rat Cardiac Sarcoplasmic Reticulum Titrated with Arachidonoyl Phosphatidylcholine
}

\author{
Tomiyasu Koyama, M.D. and Ming-Yan Zhu, M.D.
}

\begin{abstract}
SUMMARY
Sarcoplasmic reticulum vesicles were prepared from rat myocardium. The intramolecular oscillation of the phosphorylation domain of $\mathrm{Ca}^{2+}$ ATPase in control vesicles and in vesicles titrated with diarachidonoyl phosphatidylcholine was studied with a nanosecond time-resolved fluorometer. The membrane viscosity of the lipid domain was decreased by the lipid titration. The phosphorylation domain was labeled with a fluorophore, anilinonaphthylmaleimide (ANM). The time course of anisotropy decay of ANM fluorescence reflects the localized oscillation in the protein structure. The half-decay time of the anisotropy was decreased by diarachidonoyl titration from $77 \mathrm{nsec}$ in control vesicles to $66 \mathrm{nsec}$, suggesting an increase in the intramolecular oscillation. Concomitantly observed decreases in membrane viscosity and $\mathrm{Ca}^{2+}$-ATPase activity suggest that the decreased membrane viscosity destabilized the $\mathrm{Ca}^{2+}$-ATPase protein structure causing a reduction in $\mathrm{Ca}^{2+}$-ATPase activity.
\end{abstract}

\section{Key Words :}

Intramolecular oscillation Dynamic microstructure Time-resolved fluorometry

Cardiac sarcoplasmic reticulum Arachidonoyl phosphatidylcholine

\footnotetext{
CA $\mathrm{A}^{2+-A T P A S E}$ protein possesses hydrophobic and hydrophilic segments. 1 The hydrophobic segment, which binds and sequestrates intracellular $\mathrm{Ca}^{2+}$ during muscle relaxation, is maintained in the appropriate position and conformation by the lipid bilayer of the sarcoplasmic reticulum (SR) in which it is embedded. The hydrophilic segment is exposed to the cytoplasm where it is free to oscillate. It has been shown ${ }^{1)}$ that the oscillation of the phosphorylation domain increases, and $\mathrm{Ca}^{2+}$-ATPase activity decreases, when the phospholipids (PL) surrounding the hydrophobic segment of the protein are replaced with shorter phospholipids, probably because the lipid bilayer is no longer able to support the hydrophobic basement structure with the appropriate degree of tightness."
}

From the Section of Physiology, Research Institute of Applied Electricity, Hokkaido University, Sapporo, 060 Japan.

Received for publication November 25, 1991.

Accepted April 27, 1992. 
Arachidonoyl fatty acid, a precursor of prostaglandins in living tissues, is present in SR at high concentration; it constitutes 16 and $21 \%$ of the total fatty acids of skeletal muscle $S R$ in rabbits and rats, respectively. ${ }^{2,3)}$ Assuming that arachidonoyl fatty acid esterifies only the sn-2 position of the sn-3-glycerophosphate backbone, ${ }^{4)}$ about one third or more of the phospholipid species in SR would contain this acyl moiety. It has been recognized that polyunsaturated fatty acids have a role in the maintenance of $\mathrm{Ca}^{2+}$-ATPase activity but the effect of arachidonoyl fatty acid on the lipid bilayer, the intracellular compartment containing $\mathrm{Ca}^{2+}$-ATPase, remains unknown. Arachidonoyl phosphatidylcholine (PC) is longer and more unsaturated, compared with the phospholipids used in our previous study ${ }^{1}$ and its effect on the intramolecular oscillation of $\mathrm{Ca}^{2+}$-ATPase has not been examined. Although $\mathrm{Ca}^{2+}$-ATPase activity in rat skeletal muscle is reported to be relatively insensitive to alterations in the degree of unsaturation produced by different diets in in-vivo experiments, ${ }^{3 \prime}$ the complex mixture of PL molecular species present in natural membranes may have buffered the effects of unsaturation. It would be of interest, therefore, to observe the effects of enrichment of SR with arachidonoyl PC on the intramolecular motion of $\mathrm{Ca}^{2+}$-ATPase.

In the present study $\mathrm{Ca}^{2+}-\mathrm{ATP}$ ase in vesicles formed from cardiac SR was first labeled with anilinonaphthylmaleimide (ANM) whose fluorescence anisotropy is reported to represent the local motion of the phosphorylation domain of $\mathrm{Ca}^{2+}$-ATPase. ${ }^{5)}$ The vesicles were then enriched by lipid titration with diarachidonoyl $\mathrm{PC}, \operatorname{di}(20: 4) \mathrm{PC}$, in cholate solution. The fluorescence and anisotropy decay curves of ANM fluorescence were recorded with a nanosecond time-resolved fluorometer and used for the estimation of the intramolecular oscillation of the phosphorylation domain. The lipid domain of the SR was labeled with diphenylhexatriene (DPH), to provide an index of the order of lipid packing and membrane viscosity of the lipid bilayers. Previous work on the effects of ischemic reperfusion and peroxidation on mitochondrial membrane viscosity ${ }^{6}$-8) have shown the usefulness of timeresolved fluorometry in the study of the dynamic microstructure of biomembranes.

\section{Materials and Methods}

The isolation of rat cardiac SR, labeling of the lipid layers and ATPase protein with the fluorophores, DPH and ANM, and measurements of their fluorescence and ATPase activity were described in detail in a previous study. ${ }^{1)}$ 
The lipid titration in the present study was made with diarachidonoyl phosphatidylcholine (di(20:4)PC). ANM-labeled SR vesicles were mixed with a cholate solution of di (20:4)PC at a concentration of $0.25 \mathrm{mg}$ of SR protein per milliliter of sucrose phosphate buffer solution containing $1 \mathrm{mg} / \mathrm{ml}$ cholate and $1 \mathrm{mg} / \mathrm{ml} \mathrm{di(20:4)PG}$ and incubated for $15 \mathrm{~min}$. During this period, the di(20:4)PC equilibrated with the native phospholipids of the SR. The mixture was layered over a $10 \%$ sucrose solution and centrifuged at $100,000 \times \mathrm{g}$ for $40 \mathrm{~min}$. SR vesicles containing $\mathrm{di}(20: 4) \mathrm{PG}$ free of cholate sedimented at the bottom of the sucrose solution. The SR vesicles used as a control were prepared by the same procedure and mixed with cholate solution alone. These procedures were carried out at ice-cold temperature. ANM $\mathrm{Ca}^{2+}$-ATPase activity was expressed as inorganic phosphate (Pi) released by incubation of $\mathrm{SR}$ vesicle suspension $(0.36 \mathrm{mg}$ protein $/ \mathrm{ml})$ with ATP $(84 \mathrm{mMol})$ in the presence of $\mathrm{Ca}^{2+}(0.1 \mathrm{mMol})$. All measurements were made at $25^{\circ} \mathrm{C}$.

\section{Results}

$\mathrm{Ca}^{2+-A T P a s e}$ activity decreased from 5.87 in control vesicles to 4.39 $\mu \mathrm{mPi} / \mathrm{hr} / \mathrm{mg}$ protein in $\mathrm{di}(20: 4) \mathrm{PC}$ titrated vesicles.
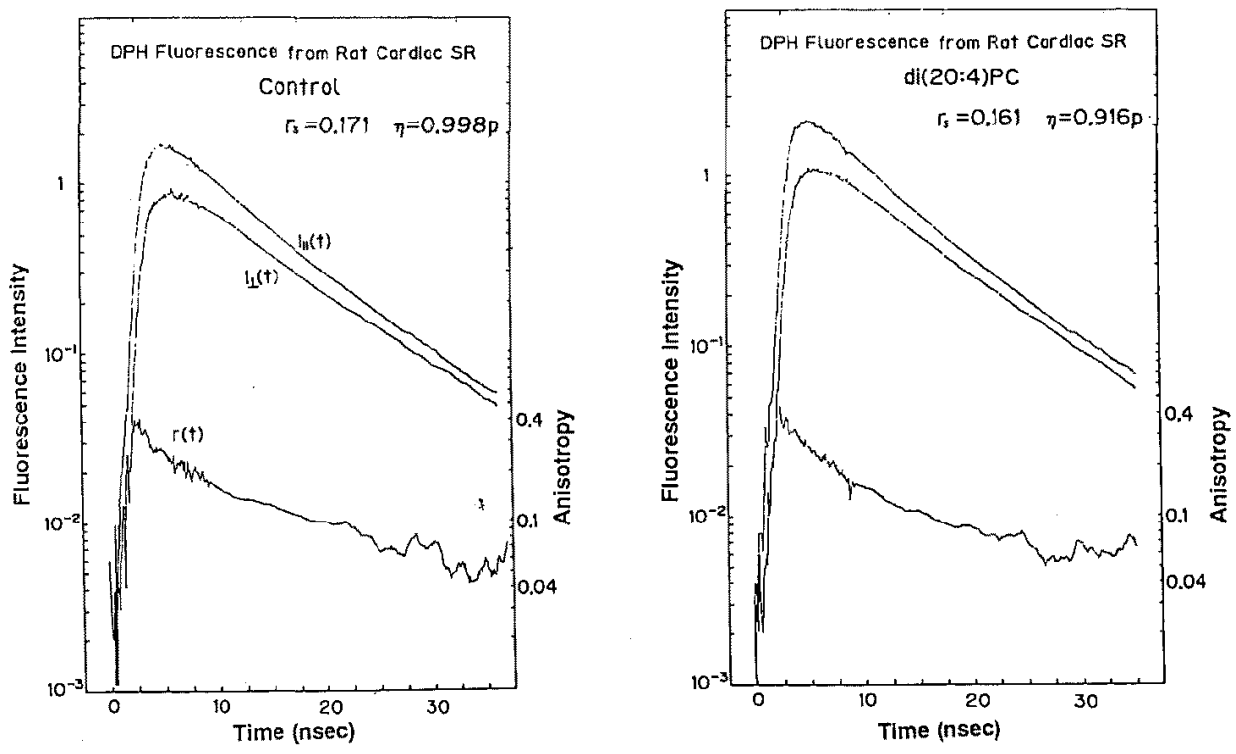

Fig. 1. DPH fluorescence $(I(t))$ and anisotropy $(r(t))$ decay curves in $\mathrm{SR}$ vesicles titrated with $\operatorname{di}(20: 4) \mathrm{PC}$. The more rapid decay of $\mathrm{DPH}$ anisotropy in di(20:4)PC-titrated SR vesicles indicates the membrane viscosity is lower than that of control $\mathrm{SR}$ vesicles. 

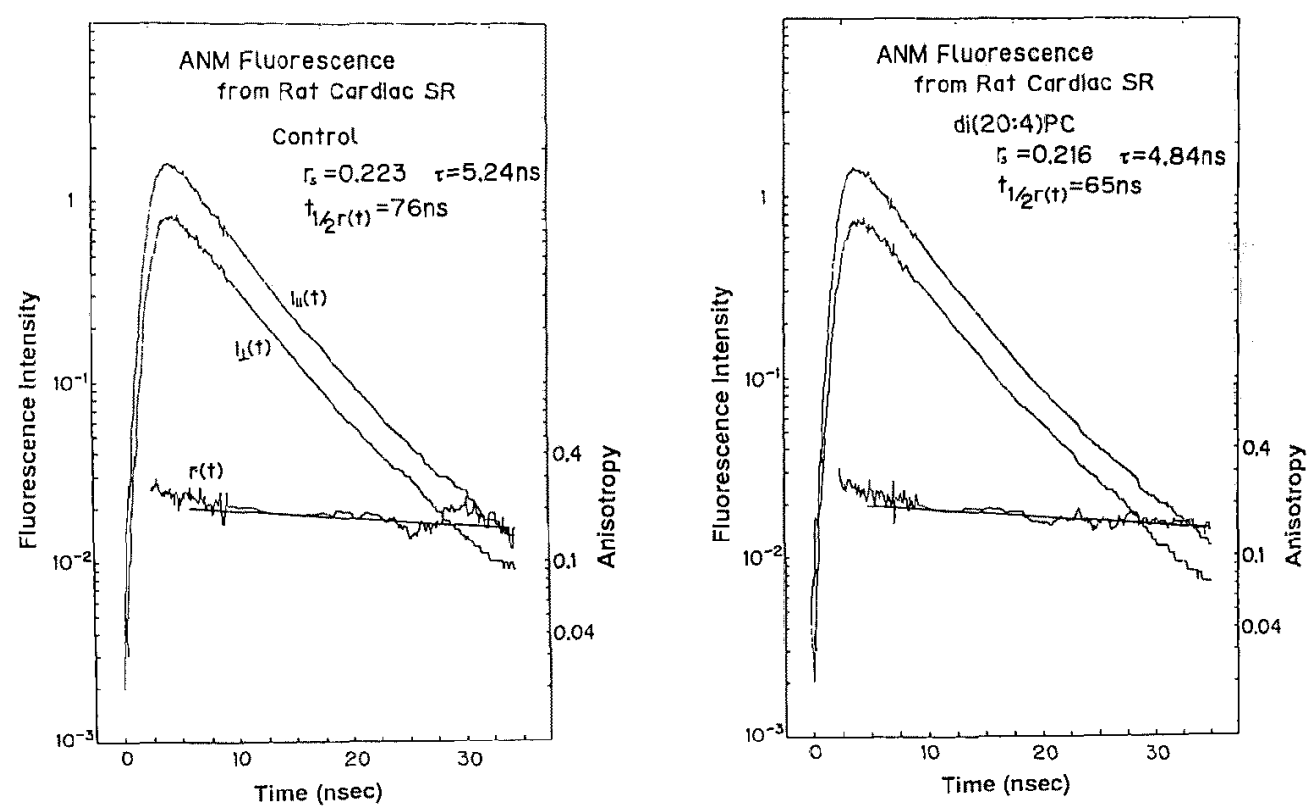

Fig. 2. ANM fluorescence and anisotropy decay curves in SR vesicles titrated with di(20:4)PC. The slow component of the anisotropy decay curve $(r(t))$ in the di(20:4)PC-titrated vesicles shows a slightly higher decay rate than that in the control vesicles.

Table I. Steady-state Anisotropy $\left(\mathbf{r}_{\mathrm{s}}\right)$, Fluorescence Life Time $(\tau)$ and Anisotropy Decay

Time $\left(t_{\frac{1}{2}} \mathrm{r}(\mathrm{t})\right)$ of Fluorescence from $\mathrm{Ca}^{2}+$-ATPase Bound ANM in SR Vesicles $(\mathbf{n}=3)$

\begin{tabular}{l|c|c}
\hline & Control & di $(20: 4) \mathrm{PC}$ \\
\hline $\mathrm{r}_{\mathrm{s}}$ & $0.223 \pm 0.002$ & $0.216 \pm 0.002$ \\
$\tau(\mathrm{nsec})$ & $5.02 \pm 0.18$ & $4.70 \pm 0.10$ \\
$\mathrm{t}_{\frac{1}{2} \mathrm{r}(\mathrm{t})}(\mathrm{nsec})$ & $77 \pm 2$ & $66 \pm 2$ \\
\hline
\end{tabular}

Mean \pm range of values.

Steady-state anisotropy of DPH fluorescence $\left(r_{s}\right)$ was smaller in di(20:4)PC-titrated vesicles than in controls. Examples of recordings of $\mathrm{DPH}$ fluorescence decay curves are shown in Fig. 1. It can be seen that the anisotropy decay rate was increased, and the membrane viscosity decreased in the di(20:4)PC-titrated vesicles as presented in the right upper corner of each graph.

ANM fluorescence and anisotropy decay curves are shown in Fig. 2. The steady-state anisotropy in the titrated vesicles was smaller than in control SR vesicles. The decay curves for ANM fluorescence and anisotropy from titrated and control vesicles are superficially similar, but the life time of 
ANM fluorescence was decreased from 5.2 to 4.8 nsec by di(20:4)PC titration. The anisotropy decay is slightly accelerated in the titrated vesicles.

As found in a previous study ${ }^{11}$ the anisotropy decay curves were not monoexponential and were analyzed as two exponential curves. The slow components, shown by straight lines in Fig. 2, were assumed to represent the time course reflecting intramolecular oscillation of the phosphorylation domain. The straight lines were extrapolated to estimate the half-decay time $\left(t_{1 / 2} r(t)\right)$. The half-decay time was $66 \pm 2 \mathrm{nsec}$ in $\operatorname{di}(20: 4)$ PC-titrated vesicles, compared with $77 \pm 2 \mathrm{nsec}$ in the control as listed in Table I.

\section{Discussion}

\section{Ca $^{2+}$-ATPase activity}

The $\mathrm{Ca}^{2+}$-ATPase activity was decreased in the $\operatorname{di}(20: 4) \mathrm{PC}$ titrated vesicles. Plotting activity against membrane viscosity together with the measurements made on vesicles titrated with the series $\operatorname{di}(n: 1) P C$ suggested a relation between enzymatic activity and membrane viscosity. This relation was probably apparent because unsaturated PLs of a single species were employed in our studies. East et al, ${ }^{91}$ who examined $\mathrm{Ca}^{2+}-\mathrm{ATPase}$ activity in vesicles reconstituted with a wide variety of phospholipid species having acyl chains of different lengths and degrees of saturation, found no correlation between the order parameter of the lipid layer of the membrane and enzymatic activity. Different PL species may affect $\mathrm{Ca}^{2+}$-ATPase activity more strongly than the order parameter which is comparable to membrane viscosity.

\section{DPH fluorescence}

The fluorescence of DPH molecules which are entrapped between the phospholipids and wobble with them reflects the packing order of PL and average membrane viscosity. ${ }^{10}$ ) A trend for the degree of order of lipid membranes to decrease with increasing polyunsaturation of phospholipids has been noted previously. ${ }^{111}$

The steady-state anisotropy of DPH fluorescence, an index of the order of the membrane phospholipids, decreased by $6 \%$ in di(20:4)PG-titrated vesicles, compared with the value in control vesicles. This decrease was accompanied by a $25 \%$ decrease in $\mathrm{Ca}^{2+}$-ATPase activity and was consistent with the finding that ATPase activity of canine skeletal muscle SR and the order parameter of its lipid membrane were reduced by the addition of diarachidonoyl $\mathrm{PG}$ to reconstituted vesicles. ${ }^{91}$

The membrane viscosity in $\operatorname{di}(20: 4)$ PC-titrated vesicles, assessed from 
the anisotropy decay curve of DPH fluorescence, was lower than in the control. These results suggested that a high proportion of PLs in the SR were replaced by di(20:4)PC and that the hydrophobic segment of $\mathrm{Ca}^{2+-A T P a s e}$ protein was surrounded by a $\operatorname{di}(20: 4) \mathrm{PC}$-enriched, less viscous lipid bilayer.

\section{ANM fluorescence}

In parallel with the decrease in $\mathrm{Ca}^{2+}$-ATPase activity and membrane viscosity, the steady-state anisotropy of ANM fluorescence decreased from a control value of 0.223 to 0.216 in $\operatorname{di}(20: 4)$ PC-titrated vesicles, suggesting a decrease in the order parameter of the phosphorylation domain of $\mathrm{Ca}^{2+}$ ATPase.

The life time of ANM fluorescence was decreased by di(20:4)PC-titration. The reduced order of the lipid domain in $\operatorname{di}(20: 4) \mathrm{PC}_{\text {-titrated vesicles }}$ may result in an easier passage of water to ANM molecules than in control vesicles. Because water molecules quench the fluorescence from hydrophobic fluorophores, the life time of ANM fluorescence is shortened. A similar alteration in ANM fluorescence decay time was observed in the previous study using PLs of shorter acyl chain length. ${ }^{11}$

The half-decay time of the ANM fluorescence anisotropy was slightly decreased in di(20:4)PG-titrated vesicles, compared with control vesicles. The decrease was not as marked as that seen previously with the shortening of the acyl chains. ${ }^{1}$

The fluorescence anisotropy is given by $r(t)=\left(I_{\| 1}(t)-I_{\perp}(t)\right) /\left(I_{\|}(t)+\right.$ $\left.2 I_{\perp}(t)\right)$, where $I_{\|}(t)$ and $I_{\perp}(t)$ represent, respectively, the parallel and perpendicular components of the fluorescence. This equation gives the difference between $I_{\|}(t)$ and $I_{\perp}(t)$ normalized for total fluorescence intensity. When ANM molecules bound to the phosphorylation domain of ATPase are illuminated with polarized pulsed light in the time-resolved fluorometer, only the molecules having their molecular axes parallel to the polarized pulsed light will absorb the light energy. When the pulse ceases, the excess energy in the ANM molecules is released as fluorescent light which is again polarized parallel to the ANM molecular axis. Accordingly, the parallel component, $I_{\|}(t)$ is initially much larger than $I_{\perp}(t)$. Then, because ANM molecules bound to the phosphorylation domain of ATPase oscillate with the intramolecular oscillation of ATPase protein, the molecular axis of ANM deviates from the original direction, causing a decrease in $I_{\|}(t)$ and an increase in $I_{\perp}(t)$. Slightly later, $I_{\perp}(t)$ also decreases, as the absorbed light energy is gradually exhausted, and the difference between $I_{\|}(t)$ and $I_{\perp}(t)$ gradually decreases. Thus, the anisotropy decay curve gives first large, and then decreasing, values according to this sequence of events. The anisotropy 


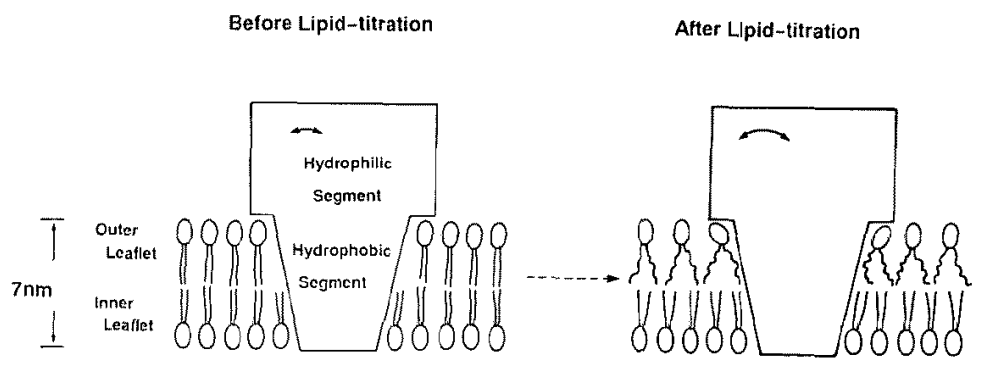

\section{Lumen of SR Vesicle}

Fig. 3. Schematic diagram of $\mathrm{Ca}_{2}{ }^{2+}$-ATPase and phospholipid bilayer of SR vesicles. This diagram was modified from a previous paper $^{\text {i' }}$ to visualize the basic idea of the present study; note the outer leafiet of the phospholipid bilayer replaced with $\operatorname{di}(20: 4) \mathrm{PC}$ which is longer and packed more loosely than the native phospholipid bilayer. The intramolecular oscillation is exaggeratedly represented with arrows.

decay, therefore, reflects the time course of alterations in the intramolecular orientation. The reduction in the half-decay time of ANM fluorescence anisotropy observed in the present study signifies a speeding-up of the alteration of orientation of the fluorophore, i.e. an increase in the intramolecular oscillation.

The avcragc carbon number and the unsaturation of PL acyl chains in skeletal muscle SR are estimated to be 18.3 and 1.9 , respectively (see Table I given by Stubbs and Kisielewski). ${ }^{3}$. The length of one unsaturated bond between carbon molecules is $1.34 \mathrm{~A}$ and its length along the long axis of the acyl chain is $1.14 \mathrm{~A}$, assuming the bond angle to be $117^{\circ}$. The length of the saturated bond is 1.54 A and its length along the long axis is $1.26 \mathrm{~A}$, assuming the bond angle to be $\left.110^{\circ} .12\right)$ The length of the acyl chain of $\operatorname{di}(20: 4) \mathrm{PC}$ is calculated to be $24.72 \mathrm{~A}$, which is longer than the $22.9 \mathrm{~A}$ length of the average acyl chain in native SR. Titration of SR vesicles with di(20:1)PC caused a decrease in ATPase activity in canine skeletal muscle SR, suggesting that the correct PL acyl chain length is necessary for high ATPase activity. ${ }^{13)}$ The effects of PLs of different chain lengths on the intramolecular motion of $\mathrm{Ca}^{2+}$-ATPase have not yet been examined in rat cardiac SR. It might be anticipated that the elongation of acyl chains would cause a reduction in the intramolecular motion, since the supporting PLs would extend further up the hydrophobic segment of the ATPase. In the present study 
with diarachidonoyl PG the intramolecular oscillation of the ATPase of rat cardiac SR increased despite the elongation of the PL. This increase in the intramolecular oscillation was probably caused by the looseness of the packing of the lipid bilayer due to the increased unsaturation of the acyl chains of the PL surrounding the hydrophobic segment of the protein. This feature is shown schematically in Fig. 3.

The titration of rat cardiac SR vesicles with di(18:1) PG tended to lengthen the half-decay time of ANM fluorescence from the control values ${ }^{11}$ probably because of the decrease in the unsaturation index. The degree of fit of the acyl chains to the molecularly rough surface of the ATPase may be important for the maintenance of ATPase activity. ${ }^{111}$ Unsaturated phospholipids may make a better fit with the surface of the ATPase, but highly unsaturated PLs may be unable to provide adequate support, resulting in an increase in the intramolecular motion of ATPase. The increased intramolecular motion may destabilize the $\mathrm{Ca}^{2+}$-ATPase protein and uncouple the enzymatic activity.

\section{REFERENGES}

1. Koyama T, Zhu M-Y: Effects of phospholipids on function and dynamic microstructure of the phosphorylation domain of sarcoplasmic reticulum from rat cardiac muscle. J Appl Cardiol 6: 229, 1991

2. Warren GB, Toon PA, Birdsall NJM, Lee AG, Metcalfe JC: Reconstitution of a calcium pump using defined membrane components. Proc Nat Acad Sci USA 71 : 622, 1974

3. Stubbs CD, Kisielewski AE: Effect of increasing the level of omega-3 fatty acids on rat skeletal muscle sarcopiasmic reticulum. Lipids 25: 553, 1990

4. Infante JP: Docosahexaenoate-containing phospholipids in sarcoplasmic reticulum and retinal photoreceptors. A proposal for a role in $\mathrm{Ca}^{2+}$-ATPase calcium transport. Mol Cell Biochem 74: 111, 1987

5. Suzuki S, Kawato S, Kouyama T, Kinosita K Jr, Ikegami A, Kawakita M: Independent flexible motion of submolecular domains of the $\mathrm{Ca}^{2+}, \mathrm{Mg}^{2+}$-ATPase of sarcoplasmic reticulum measured by time-resolved fluorescence depolarization of site-specially attached probes. Biochem 28: 7734,1989

6. Koyama T, Zbu M-Y, Kinjo M, Araiso T: Protective effects of idebenone against alterations in dynamic microstructure induced by lipid peroxidation in rat cardiac mitochondria. Jpn Heart J 32: 91, 1991

7. Koyama T, Zhu M-Y, Kinjo M, Araiso T: Dynamic microstructure of mitochondrial membranes from rabbit heart subjected to reperfusion after ischemia. Jpn Heart J 32: 247, 1991

8. Koyama T, Zhu M-Y, Kinjo M, Araiso T: Dynamic microstructure and hydration of peroxidized membrane of rat cardiac mitochondria and effects of adriamycin. Jpn J Physiol 40 : 635,1990

9. East JM, Jones OT, Simmonds AC, Lee AG: Merabrane fluidity is not an important physiological regulator of the $\left(\mathrm{Ca}^{2+}-\mathrm{Mg}^{2+}\right)$-dependent ATPase of sarcoplasmic reticulum. J Biol Chem $259: 8070,1984$

10. Araiso T, Koyama T: Picosecond fluorescence study of the cone angle of the wobbling motion of rod-like fluorophore in lipid bilayers. Chem Phys Lipids 50: 105, 1989

11. Stubbs CD, Kouyama T, Kinosita K Jr, Ikegami A: Effect of double bonds on the dynamic 
properties of the hydrocarbon region of lecithin bilayers. Biochem 20:4257, 1981

12. Roberts JD, Caserino M: Basic Principles of Organic Chemistry, WA Benjamin Inc, NY, 1964

13. Johannson A, Keightley CA, Smith GA, Richards CD, Hesketh TR, Metcalfe JC: The effect of bilayer thickness and $\mathrm{n}$-alkanes on the activity of the $\left(\mathrm{Ca}^{2+}+\mathrm{Mg}^{2+}\right)$-dependent ATPase of sarcoplasmic reticulum. J Biol Chem 256: 1643, 198 I 